In der Rubrik „Literatur kompakt" werden die wichtigsten Originalarbeiten aus der internationalen Fachliteratur referiert.

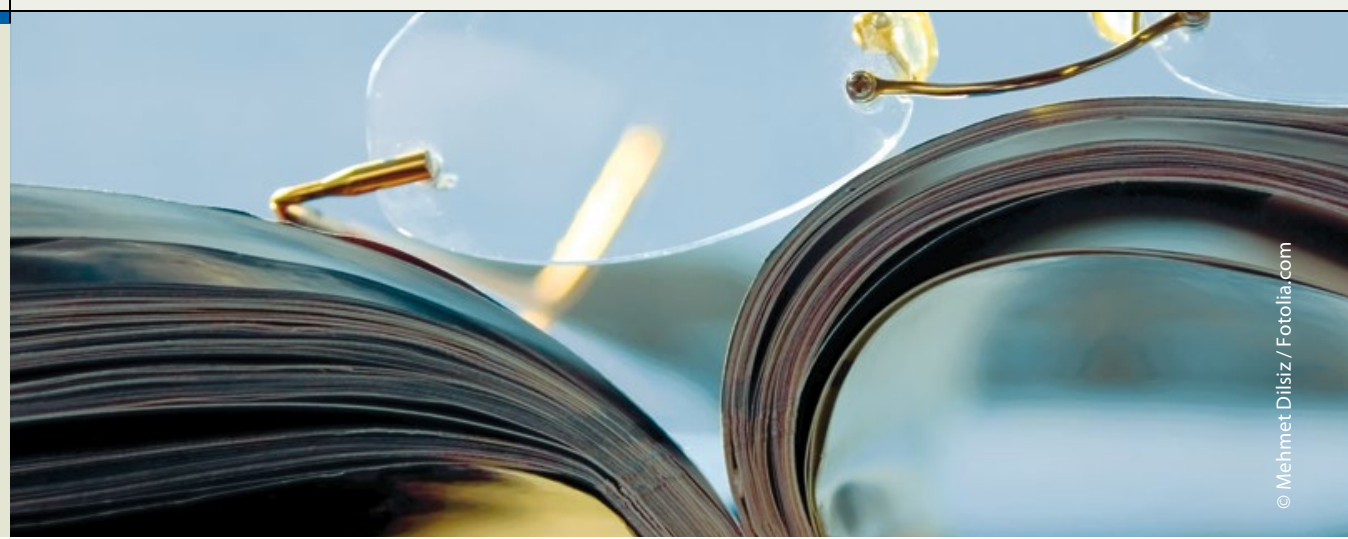

\section{Antikörper wirkt bei hartnäckiger Neurodermitis}

\author{
Inwieweit der monoklonale Antikörper Dupilumab in der Lage ist, \\ hartnäckige Neurodermitis-Symptome zu bessern, wurde in einer \\ Dosisfindungsstudie getestet.
}

$\mathrm{D}$ upilumab ist ein monoklonaler Antikörper, der am Interleukin-4-Rezeptor $\alpha$ angreift. Damit blockiert er die Aktivierung der Interleukine 4 und 13, die in der Pathogenese der atopischen Dermatitis eine wichtige Rolle spielen.

Wirksamkeit und Sicherheit mehrerer Dupilumab-Regime bei Neurodermitis wurden jetzt in einer randomisierten, kontrollierten Phase-IIb-Studie getestet. Bei den Teilnehmern handelte es sich um erwachsene Neurodermitispatienten aus sieben verschiedenen Nationen, darunter Deutschland. Sie alle litten unter mittelgradigen bis schweren Symptomen, die auf eine topische Therapie innerhalb eines halben Jahres nur unzureichend angesprochen hatten. $\mathrm{Zu}$

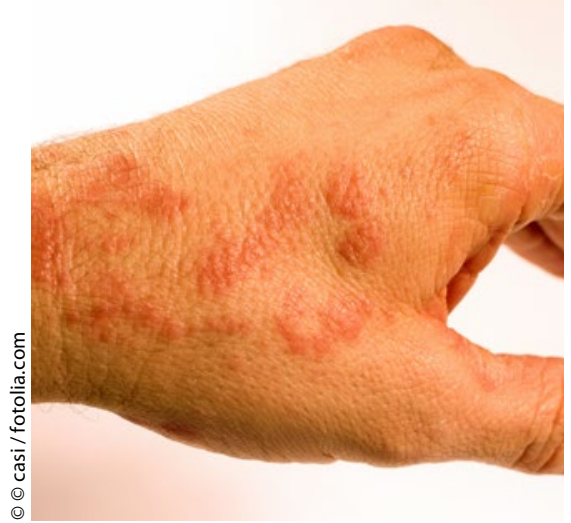

Hoffnung für Patienten mit atopischem Ekzem?
Studienbeginn lag der EASI (Eczema Area and Severity Index), der das Ausmaß und die Schwere des Ekzems misst, bei mindestens 12 (von maximal 72) und der IGA-Score (Investigator's Global Assessment) bei mindestens 3 (von 5) Punkten. Die Hautveränderungen betrafen $10 \%$ oder mehr der gesamten Hautoberfläche.

Die 379 Patienten wurden nach dem Zufallsprinzip in sechs Gruppen eingeteilt: Fünf erhielten unterschiedliche Antikörper-Regime, jeweils subkutan. Die sechste Gruppe (61 Patienten) bekam ein Placebo gespritzt. Als primären Endpunkt hatten die Forscher die prozentuale Veränderung im EASI-Score innerhalb von 16 Wochen nach Therapiebeginn definiert.

In nahezu allen Verumgruppen zeigte sich eine deutliche dosisabhängige Verbesserung im Vergleich zu Placebo. Die Ergebnisse für die einzelnen Regime: _300 mg einmal die Woche: $-73,7 \%$ -300 mg alle zwei Wochen: $-68,2 \%$ _200 mg alle zwei Wochen: $-65,4 \%$ -300 mg alle vier Wochen: $-63,5 \%$ - $100 \mathrm{mg}$ alle vier Wochen: $-44,8 \%$. Unter Placebo gingen die Werte im EASI-Score im Mittel um 18,1 \% zurück. Damit waren die Unterschiede zu Placebo für alle 300- und 200-mg-Regime signifikant; lediglich das 100-mg-Regime erzielte kein signifikantes Ergebnis.

Deutlich mehr Patienten erreichten unter Dupilumab einen IGA-Wert von
0 oder 1, was einer (fast) ekzemfreien Haut entspricht. Und auch im SCORAD-Score (SCORing Atopic Dermatitis), der Intensität und Ausmaß der Hautveränderungen misst, waren die Verumgruppen jeweils Placebo deutlich überlegen, mit Ausnahme der 100-mgGruppe. Signifikante Verbesserungen ergaben sich (außer bei $100 \mathrm{mg}$ ) auch bei den subjektiven Kriterien Juckreiz (Pruritus Numeric Rating Scale) und Lebensqualität (Dermatology Life Quality Index).

In den meisten Fällen hatte sich die Krankheitsaktivität bereits nach einer Woche deutlich gebessert. Obwohl der Antikörper insgesamt gut toleriert wurde, brachen 21 Patienten die Therapie vorzeitig ab. Zehn von ihnen stammten aus der 100-mg-Gruppe. Auffällig waren 13 Fälle mit Lippenherpes, von denen sich die meisten ebenfalls in dieser Gruppe fanden, und ein Asthmafall.

Fazit: Wie die Phase-IIb-Studie nahelegt, lassen sich mittelschwere bis schwere Neurodermitis-Symptome durch den Antikörper Dupilumab signifikant bessern. Insbesondere der Juckreiz ließ sich bei ausreichender Dosierung gut unter Kontrolle bringen. Weitere Studien müssen unter anderem klären, ob die Wirkung und Sicherheit auch bei längerer Anwendung und in Kombination mit einer Lokaltherapie erhalten bleibt und was es mit dem Lippenherpes auf sich hat.

Dr. Elke Oberhofer

Thaçi D et al. Efficacy and safety of dupilumab in adults with moderate-to-severe atopic dermatitis inadequately controlled by topical treatments: a randomised, placebo-controlled, dose-ranging phase $2 \mathrm{~b}$ trial. Lancet 2015; doi: 10.1016/S0140-6736(15)00388-8 